\title{
Digital photonic production of 3D micro fluidics in glass by high speed micro scanner
}

\author{
Jens Gottmann ${ }^{1,2}$, Martin Hermans ${ }^{1,2}$, Jürgen Ortmann ${ }^{1,2}$ \\ ${ }^{1}$ Lehrstuhl für Lasertechnik, RWTH Aachen University, Steinbachstraße 15, 52074 Aachen, Germany \\ ${ }^{2}$ LightFab (in the course of incorporation) Steinbachstr. 15, 52074 Aachen, Germany \\ Author e-mail address: jens.gottmann@llt.rwth-aachen.de
}

\begin{abstract}
Digital photonic production of 3D microfluidic devices and assembled micro mechanics inside fused silica glass is carried out using In-volume Selective Laser-induced Etching (ISLE). From CAD data of digital 3D objects the trajectories of the laser focus are automatically generated. The CAM software has been developed controlling different fs- and ps-lasers, the beam deflection systems and the translation stages to produce the structures automatically.
\end{abstract}

\section{Introduction}

Digital photonic production (DPP) is the production of parts directly from digital CAD data using laser radiation. For computer-aided manufacturing (CAM) software generates trajectories to be scanned by the laser radiation from the 3D CAD data describing the part to be produced and controls the positioning systems and the laser accordingly. Because in digital photonic production all information needed for the production of the part is digital and neither mold nor mask are needed, the production cost is rather independent from lot size enabling individualized production. Since the in-volume selective laser-induced etching process (ISLE) enables digital photonic production of 3D parts in transparent materials like glasses and sapphire, the above mentioned advantages are used for the production of microfluidic devices for applications e.g. in medical diagnosis. ISLE of micro channels in fused silica glass and sapphire is a fabrication process under investigation for more than eight years now [1-4]. Special microscanners with CAM software have been developed [5] and versatile table-top machining centers are in development [6]. Enabled by newly developed high average power femtosecond lasers $(150-500 \mathrm{~W})$ and high speed micro scanners ( $>10 \mathrm{~m} / \mathrm{s}$ at $2 \mu \mathrm{m}$ focus) efficient digital photonic production of complex micro structured parts is possible using ISLE [7].

\section{High speed micro scanner}

Two types of modular high speed micro scanners have been developed: A flexible system for 3D micro structuring inside transparent materials has been developed to be mounted on the z-axis of a commercial translation stage (Fig. 1, left). This micro scanner should be matched with fs- or ps-lasers with repetition rate $100 \mathrm{kHz}-5 \mathrm{MHz}$ and average power of $1-50 \mathrm{~W}$ and is capable to write arbitrary structures with $>0.2 \mathrm{~m} / \mathrm{s}$ with a focus radius of $1 \mu \mathrm{m}$ at $1 \mu \mathrm{m}$ wavelength and to produce several complex 3D prototypes per day. The second type is a high speed micro scanner optimized for high throughput of special structures, e.g. micro channels. The high speed micro scanner is designed for fs- or ps-lasers with repetition rate 5-100 MHz and average power of 20-150 $\mathrm{W}$ and is capable to write structures with $10 \mathrm{~m} / \mathrm{s}$ on a $500 \mu \mathrm{m}$ track radius with a focus radius of $1 \mu \mathrm{m}$ at $1 \mu \mathrm{m}$ wavelength. Included are a fast focusing module, a 2-axis translation stage and a microscope for inspection and alignment (Fig. 1, right)
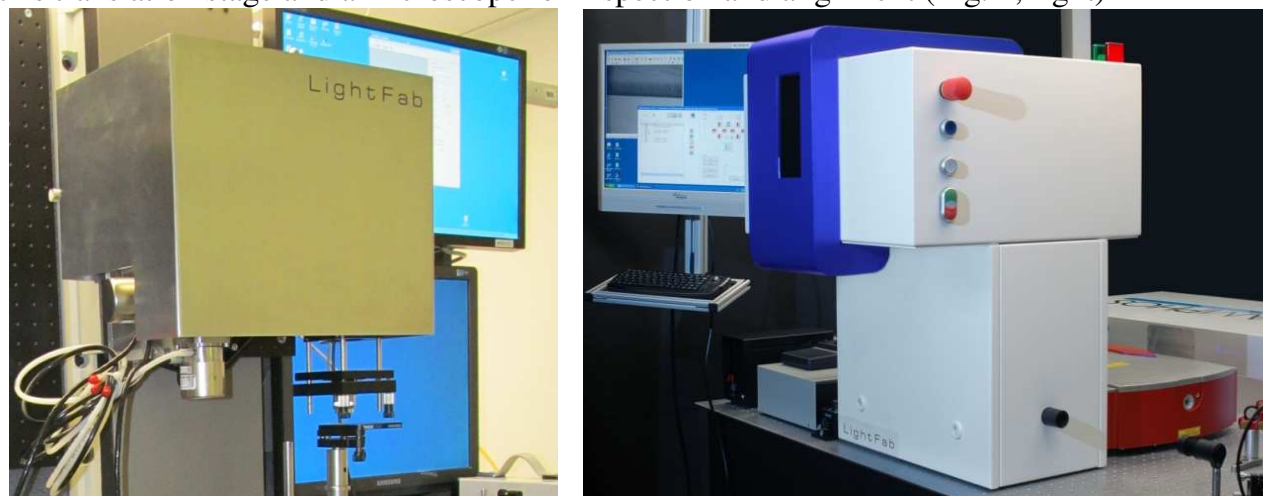

Fig. 1. Modular high speed micro scanner systems: The flexible micro scanner prototype mounted on the z-axis of a 3-axis translation stage (left) and the high throughput micro scanner with translation stage included behind the blue sliding door (right) 


\section{In-volume selective laser-induced etching of 3D micro parts in fused silica}

In-volume selective laser-induced etching (ISLE) is a two-step process: In the first step material transparent to the laser radiation is modified locally inside the volume by ultra-short pulsed laser radiation focused with NA of 0.3 . A connected volume inside the material is modified by scanning of repetitive pulses, which must have at least one contact to the surface of the work piece. For digital production of 3D microstructures from the CAD data a set of stacked 2D scanning trajectories are computed (slicing; Fig. 2 left), which are fed automatically from bottom to top to the CAM software controlling the translation stages, the modules of the scanning system and the laser power synchronously. If the structure to be produced is larger than the scanning field of the lens, the 2D trajectories are automatically divided in adjacent tiles and are scanned and stepped subsequently (Fig. 2 center).
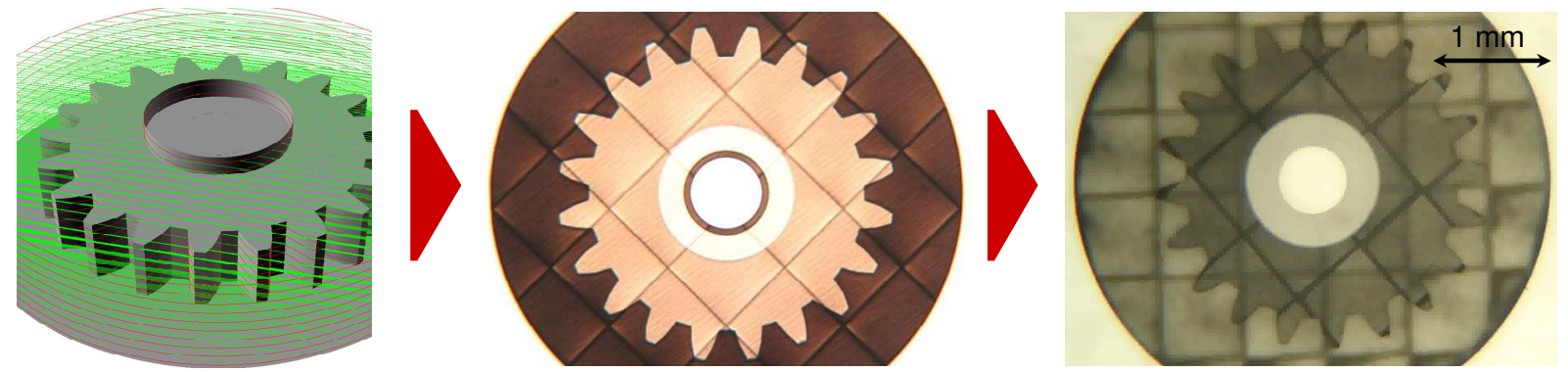

Fig. 2. Laser trajectories automatically generated from 3D CAD file (schematic, left), micrograph of modified structure (center) and resulting microstructure after etching the modified volume selectively (right).

In the second step the work piece is exposed to aqueous solution of potassium hydroxide (KOH). Because the material, modified by the laser radiation, is etched much faster than the unmodified material, the modified volume is selectively removed resulting in hollow structures inside the work piece. The freely rotatable gear is obtained already mounted on its axis (Fig. 2, right and Fig. 3, left). In the same way a 3D micro channel for medical diagnosis is directly produced from CAD data consisting of a buried flat channel, tapered inlets and connectors for flexible tubes (Fig. 3, right). The rotatable gear has also been produced inside the buried flat channel.
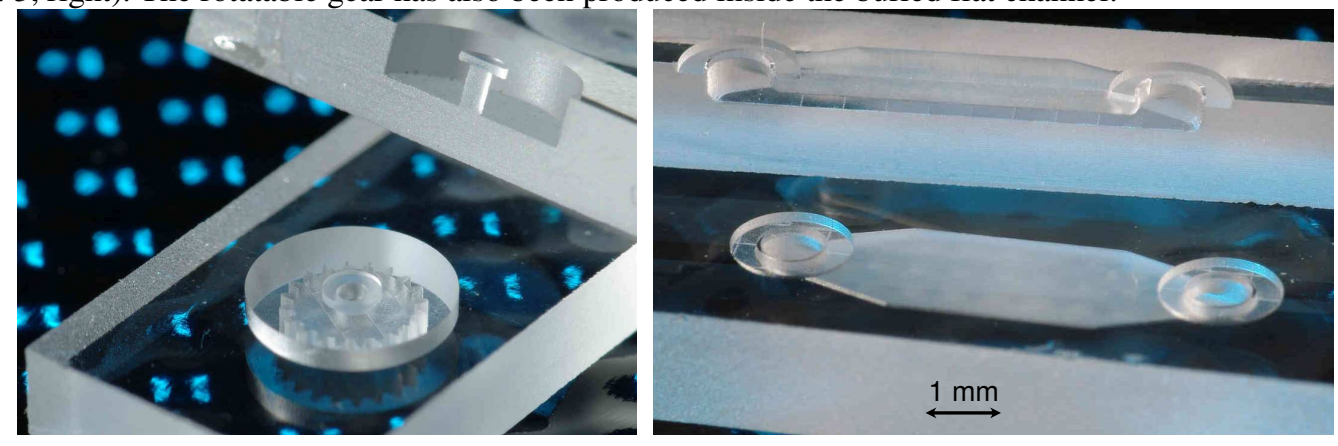

Fig. 3. Free rotating gear produced directly from CAD data already assembled on its shaft (top-view and cross-section, left) and 3D micro fluidic device in $2 \mathrm{~mm}$ thick fused silica made by ISLE directly from CAD file (top-view and cross-section, right)

\section{Acknowledgement and References}

The work is funded by NRW.Transfer Science-to-Business PreSeed to prepare the spin-off LightFab making both the micro scanners and the 3D micro structured glass parts commercially available.

[1] Y. Bellouard, A. Said, M. Dugan, P. Bado, "Fabrication of high-aspect ratio micro fluidic channels and tunnels using femtosecond laser pulses and chemical etching," Optics Express 12, 2120 (2004).

[2] C. Hnatovsky, R. S. Taylor, E. Simova, V. R. Bhardwaj, D. M. Rayner, P. B. Corkum, "Polarization-selective etching in femtosecond laserassisted microfluidic channel fabrication in fused silica," Optics Letters 30, 1867-1869 (2005).

[3] S. Matsuo, Y. Shickijo, T. Tomita, S. Hashimoto, “Laser fabrication of ship-in-a-bottle microstructures in sapphire,” JLMN 2, 114 (2007).

[4] D. Wortmann, J. Gottmann, N. Brandt, H. Horn-Solle, "Micro- and nanostructures inside sapphire by fs-laser irradiation and selective etching," Optics Express 16, 1517-1522 (2008).

[5] J. Gottmann, M. Hörstmann-Jungemann, M. Hermans, D. Beckmann, "High speed and high precision fs-laser writing using a scanner with large numerical aperture," JLMN 4, 192-196 (2009).

[6] Y. Bellouard, A. Champion, B. Lenssen, M. Matteucci, A. Schaap, M. Beresna et. al., "The femtoprint project,” JLMN 7, 1-10 (2012).

[7] J. Gottmann, M. Hermans, J. Ortmann, "Digital photonic production of micro structures in glass by in-volume selective laser-induced etching using a high speed micro scanner,” Physics Procedia 39, 534 - 541 (2012). 